\title{
PEANO CURVES IN FUNCTION ALGEBRAS
}

\author{
LARRY Q. EIFLER
}

Abstract. We give a short proof of the following result which was obtained by Pełczyński. If $X$ is an uncountable, compact metric space and if $A$ is a function algebra on $X$, then there exists $f$ in $A$ such that $f(X)$ has interior in the plane.

Let $X$ be a compact metric space and let $A$ be a function algebra on $X$. If one restricts the "size" of $f(X)$ for each $f$ in $A$, then the space $X$ is likewise restricted. For example, Rudin [4] has shown that $f(X)$ is countable for each $f$ in $A$ if and only if $X$ is countable. Of course, $f(X)$ is countable for each $f$ in $A$ implies $A=C(X)$. Pełczyński [2] has shown that $X$ is uncountable if and only if $A$ contains a closed subspace $M$ and $X$ contains a perfect, closed subset $K$ such that $f \rightarrow f \mid K$ is an isometry of $M$ onto $C(K)$. Our purpose is to give a different approach to some of Pełczyński's work.

If $X$ is a compact subset of the plane $C$, then $P(X)$ denotes the uniform closure in $C(X)$ of the polynomials. We begin with the following result.

Theorem. Suppose $X$ is an uncountable, compact subset of $C$. There is a closed, uncountable subset $K$ of $X$ such that $K$ is a peak set for $P(X)$ and $P(X) \mid K=C(K)$.

Proof. We may assume that $X$ is polynomially convex. By Wermer's characterization [6] of the annihilator of $P(X)$, there is a nonnegative measure $\mu$ on $\partial X$, the boundary of $X$, such that $v \perp P(X)$ and $v$ is supported on $\partial X$ implies that $\nu$ is absolutely continuous with respect to $\mu$. Hence, by Bishop's generalization [1] of the Rudin-Carleson theorem, we only need to find $K \subseteq \partial X$ such that $\mu(K)=0$ and $K$ is closed and uncountable. Such a $K$ exists. Namely, since $\partial X$ is uncountable, there is a continuous map $g$ on $\partial X$ onto $[0,1] \times[0,1]$. Then $\mu\left(g^{-1}(\{x\} \times[0,1])\right)=0$ for all but countably many $x$ in $[0,1]$.

Corollary. Suppose $Y$ is a compact metric space and $A$ is a function algebra on $Y$. If $f(Y)$ has no interior in $C$ for each $f$ in $A$, then $Y$ is countable and hence $A=C(Y)$.

Received by the editors June 15, 1971 and, in revised form, September 13, 1971. AMS 1970 subject classifications. Primary 46J10.

Key words and phrases. Function algebras. 
Proof. Suppose $f(Y)=X$ is uncountable for some $f$ in $A$. Then by the above result there is $g \in P(X)$ such that $g(X)$ has interior in $C$. Hence, $(g \circ f)(Y)=g(X)$ has interior in $C$ and $g \circ f$ belongs to $A$ which is a contradiction. Therefore, $f(X)$ is countable for each $f$ in $A$ and Rudin's result [4] implies $Y$ is countable.

Note. Much more can be said in the case of the disc algebra, the algebra of continuous functions on the unit circle $T$ which have analytic extensions to the open unit disc. For example ([3], [5]), there is a function of the form $f(z)=\sum_{n=1}^{\infty} a_{n} z^{n}$ where $\sum_{n=1}^{\infty} a_{n} z^{n}$ converges absolutely on $T$ and $f(T)$ has interior in the plane.

\section{REFERENCES}

1. E. Bishop, A general Rudin-Carleson theorem, Proc. Amer. Math. Soc. 13 (1962), 140-143. MR 24 \#A3293.

2. A. Pełczyński, Some linear topological properties of separable function algebras, Proc. Amer. Math. Soc. 18 (1967), 652-660. MR 35 \#4737.

3. G. Piranian, C. J. Titus and G. S. Young, Conformal mappings and Peano curves, Michigan Math. J. 1 (1952), 69-72. MR 14, 262.

4. W. Rudin, Continuous functions on compact spaces without perfect subsets, Proc. Amer. Math. Soc. 8 (1957), 39-42. MR 19, 46.

5. R. Salem and A. Zygmund, Lacunary power series and Peano curves, Duke Math. J. 12 (1945), 569-578. MR 7, 378.

6. J. Wermer, Seminar über Funktionen-Algebren, Lecture Notes in Math., no. 1, Springer-Verlag, Berlin, 1964. MR 35 \#5947.

Department of Mathematics, louisiana State University, Baton Rouge, LOUISIANA 70803 